\title{
Development of microsatellite markers for the critically endangered conifer Glyptostrobus pensi- lis (Cupressaceae) using transcriptome data
}

\author{
Xin-Yu Li ${ }^{1}$, Xue-Ying Lin', Markus Ruhsam², Lu Chen ${ }^{1}$, Xing-Tong Wu' ${ }^{1}$, Min-qiu Wang ${ }^{1}$, Philip I. \\ Thomas $^{2}$ and Ya-Feng Wen ${ }^{1 *}$
}

\author{
${ }^{1}$ Central South University of Forestry and Technology, Changsha, Hunan, 410004, China. \\ ${ }^{2}$ Royal Botanic Garden Edinburgh, 20 A Inverleith Row, Edinburgh EH3 5LR, United Kingdom. \\ * Corresponding author:Ya-Feng Wen, E-mail: wenyafeng7107@163.com
}

\begin{abstract}
Glyptostrobus pensilis (Cupressaceae) is a critically endangered conifer which occurs in China, Laos, and Vietnam where it is only known from a few populations. Here we aim to develop microsatellite markers which can be used to study the genetic variation within this species. Using transcriptome data we tested 170 SSR loci for polymorphism in 16 samples. Twenty-three loci were polymorphic and selected for the genetic analysis of 83 individuals from three Chinese populations. The number of alleles per locus and population ranged from one to eight, the observed and expected heterozygosity from $H_{o}=0.00-1.00$ and $H_{e}=0.00-0.83$, respectively. Fifteen loci deviated from Hardy-Weinberg equilibrium in at least one of the three populations. The majority of loci could also be successfully amplified in four related species, namely Cryptomeria fortunei, Taxodium distichum, Taxodium ascendens and Cunninghamia lanceolata. These developed microsatellites are suitable for population genetic studies of Glyptostrobus pensilis and related species.
\end{abstract}

Keywords: : Glyptostrobus pensilis; next-generation sequencing; Expressed Sequence Tag (EST); simple sequence repeat (SSR) markers

\section{Introduction}

Glyptostrobus pensilis (Staunt.) Koch is a monotypic genus in the conifer family Cupressaceae. In China, its distribution is centered in the Pearl River delta (Guangdong Province), the central region of Fujian Province, and the northeast part of Jiangxi Province (Li and Xia, 2004). A few wild populations have also been found in Vietnam and Laos, extending its latitudinal distribution from $28^{\circ} \mathrm{N}$ to $13^{\circ} \mathrm{N}$ (Averyanov et al., 2009; Thomas and LePage, 2011). The fossil record shows that $G$. pensilis became extinct in northeastern China and adjoining areas possibly during the early Pleistocene (LePage, 2007). This species was widely distributed in Guangzhou, China until the late Holeocene (Xu and Li, 1959), however, due to widespread cultivation over several centuries, the true natural distribution remains uncertain (Yu, 1995; Li and Xia, 2005). During the Holocene, and especially over the last two millennia, the riparian and flood plain habitats of $G$. pensilis have been seriously impacted by human activities, especially as a result of the development of agriculture and rice cultivation. This has led to high individual mortality and the rapid decline of most $G$. pensilis populations (Li and Xia, 2004; Li and Xia, 2005; Nguyen et al., 2013). Natural regeneration is extremely rare, probably due to low seed viability and loss of suitable habitat. Currently, G. pensilis is evaluated as Critically Endangered (CR) on the IUCN Red List of Threatened Species (Thomas et al., 2011).

Simple sequence repeats (SSR) are co-dominant and highly polymorphic markers which can be developed cheaply and 
efficiently using next-generation sequencing technology (Simon et al., 2009). Previous molecular studies on Glyptostrobus using chloroplast microsatellites (cpSSR) (Nguyen et al., 2013) and ISSR markers showed that genetic diversity was remarkably low (Li and Xia, 2005; Wu, 2011). However, these studies only focused on a limited part of the distribution range of this conifer and to date, there have been no range wide studies of its genetic diversity and structure or its phylogeographic history. Recently, Wang et al (2019) developed 10 polymorphic microsatellite markers for this species using restriction site-associated DNA sequencing (RAD-Seq). In this study, we developed 23 Expressed Sequence Tag-Simple Sequence Repeat (EST-SSR) markers for G. pensilis using transcriptome data, which can be used to investigate its range wide genetic structure and phylogeography. Additionally, we tested the transferability of the loci in four related species: Cryptomeria fortunei, Taxodium distichum, Taxodium ascendens, Cunninghamia lanceolata.

\section{Materials and Methods}

\section{Microsatellite detection}

Fresh, young stems and leaf tissue of G. pensilis were collected from a plantation in Hunan Province $\left(28^{\circ} 8^{\prime} 16.48^{\prime \prime} \mathrm{N}\right.$, $112^{\circ} 59^{\prime} 28.36^{\prime \prime} \mathrm{E}, 90 \mathrm{~m}$ ) and were immediately frozen in liquid nitrogen for RNA extraction. Total RNA was extracted using the RNAprep Pure plant kit (Tiangen) following the manufacturer's instructions. Qubit Flourometer and Agilent2100 were used to assess the concentration and the purity of the extracted RNA which was then used to construct a CDNA library and sequenced on a Illumina Genome analyzer HiSeq 2500 platform. Raw data were assembled de-novo using the software Trinity (http://trinityrnaseq.sourceforge.net/). The software MISA (http://pgrc.ipkgatersleben.de/misa/) was used to detect SSR motifs with 2, 3, 4, 5 and 6 bp nucleotide repeats. Primer3plus (http://www.bioinformatics.nl/cgi-bin/primer3plus/primer3plus.cgi) was used for primer design with the following parameters: primer length $18-27 \mathrm{bp}$, annealing temperature $57-63^{\circ} \mathrm{C}$, GC $\%$ content $20-80 \%$, and expected product length range of 100-300 bp; continuous A or T were not allowed in the 3 ' end. Loci with a minimum number of 8 repeats were selected for amplification as they may have higher polymorphisms (Ueno et al., 2012). In total, 170 SSR loci were selected and evaluated for their suitability using $16 \mathrm{G}$. pensilis DNA samples (6 samples from DM, 5 from PNSL, and 5 from GZHN).

\section{Microsatellite screening}

Whole genomic DNA was extracted from dried leaves using the CTAB (cetyltrimethylammonium bromide) method (Tsumura et al., 1995). PCR amplification was carried out in volumes of $20 \mu \mathrm{l}$ using the following protocol: $10 \mu \mathrm{L}$ of $2 \times$ Taq PCR Master Mix (constituent: $20 \mathrm{mM}$ Tris- $\mathrm{HCl}, 100 \mathrm{mM} \mathrm{KCl,} 3 \mathrm{mM} \mathrm{MgCl}{ }_{2^{\prime}}$ $0.1 \mathrm{U}$ Taq Polymerase/ $\mu \mathrm{L}, 500 \mu \mathrm{M}$ dNTP each; Tiangen, Beijing, China), $1 \mu \mathrm{l}$ forward primer $(10 \mu \mathrm{M})$ and $1 \mu \mathrm{l}$ reverse primer (10 $\mu \mathrm{M}), 3 \mu \mathrm{l}$ of $20-50 \mathrm{ng} / \mu \mathrm{l}$ DNA template, and $5 \mu \mathrm{l}$ of ddH2O. The mixture was then cycled through the profile: $94^{\circ} \mathrm{C}$ for $4 \mathrm{~min} ; 10$ cycles of $94^{\circ} \mathrm{C}$ for $40 \mathrm{~s}, 65^{\circ} \mathrm{C}$ for $35 \mathrm{~s}$ (decreased by $1^{\circ} \mathrm{C}$ every cycle), $72^{\circ} \mathrm{C}$ for $30 \mathrm{~s}$, followed by 32 cycles of $94^{\circ} \mathrm{C}$ for $40 \mathrm{~s}, 57^{\circ} \mathrm{C}$ for $35 \mathrm{~s}, 72^{\circ} \mathrm{C}$ for $30 \mathrm{~s}$ with a final extension at $60^{\circ} \mathrm{C}$ for $30 \mathrm{~min}$. PCR products were visualized on a $2 \%$ agarose gel. All loci which could be amplified successfully were tested individually using $16 \mathrm{G}$. pensilis samples to establish their polymorphic nature. For this second round of amplifications we used fluorescently labelled primers (FAM, HEX, TAMRA and ROX, Applied Biosystems) applying the same PCR protocol as above. PCR products were run on an Applied Biosystems 3130 Genetic Analyzer ( $A B \mid 3130)$ adding a LIZ-500 labeled internal size standard (Applied Biosystems, Foster City, California, USA) to size fragments. The data were analyzed using the software Gene Marker V1.80. Among 170 SSR loci, 130 loci could be amplified successfully but only 23 loci were polymorphic and produced clear and interpretable peaks. The sequences of these 23 loci were deposited in the NCBI database (Table1) and used subsequently to investigate the genetic diversity of three Chinese $G$. pensilis populations (Appendix 1).

\section{$\underline{\text { Table1 }}$}

Characterization of 23 microsatellite loci developed in Glyptostrobus pensilis.

\begin{tabular}{|c|c|c|c|c|c|c|}
\hline Locus" & $\begin{array}{c}\text { Repeat } \\
\text { motif }\end{array}$ & Primer sequences (5'-3') & $\begin{array}{c}\text { Size } \\
\text { (bp) }\end{array}$ & $\begin{array}{c}\text { Gennanank } \\
\text { aceession } \\
\text { no. }\end{array}$ & Putative function & $\begin{array}{c}\text { E- } \\
\text { value }\end{array}$ \\
\hline Uniggne_6272 & $(\mathrm{AG})_{11}$ & $F$ :FAM)TGGAGACCATTACGCGTTCA & 287 & MH061285 & $\begin{array}{l}\text { mRNA,clone:CFFL } \\
\text { 040_E19[Cryptomer }\end{array}$ & 0.000 \\
\hline Unigene_24035 & $(\mathrm{AG})_{\mathrm{B}}$ & $\begin{array}{l}\text { R: AAGAGGGGCATGTACGCTTC } \\
\text { F: (HEX)CATTTCCCCCGGCAGATCAT } \\
R: \text { GCCAAACATACAGCAGGTGC }\end{array}$ & 253 & Мно61287 & 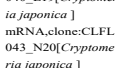 & 0.000 \\
\hline Unigene_1 15398 & $(\mathrm{AT}) \|$ & $\begin{array}{l}\text { F: (TAMRA)CTGTTCCCCTGTGCATCATA } \\
\text { R: AGATCGTGAGGGTTCGAGTC }\end{array}$ & 307 & MH158506 & 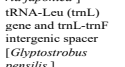 & $1 \mathrm{e}-154$ \\
\hline Unigene_622277 & $(\mathrm{TA})_{0}$ & $\begin{array}{l}\text { F: (FAM)AGTATCCCGAGGGTGAATACA } \\
R: \text { TAGCCGATGATCCTCGCATG }\end{array}$ & 163 & мно661297 & 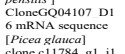 & $4 c-78$ \\
\hline Unigenc_35432 & $(\mathrm{TG})_{8}$ & $\begin{array}{l}F:(\text { HEX)TCGCGATTCTGAGATCCAGC } \\
R: \text { CGGCCACCAAATCCTTCTCT }\end{array}$ & 209 & мH061291 & 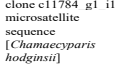 & $6 c-105$ \\
\hline Unigene__3959 & 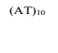 & $\begin{array}{l}F: \text { (TAMRA)TTCCTGATACCCACAAGCAT } \\
R: \text { CAATTGAAATCAACCAAGTGGA }\end{array}$ & 111 & MH158507 & 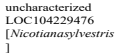 & $2 \mathrm{e}-05$ \\
\hline Unigene_51014 & $(\mathrm{AG})_{9}$ & $\begin{array}{l}\text { F: (FAM)AGAGATGTGGGTATGGAGTTCT } \\
R: \text { :CCATCCCTCTCTATCTCCCTAAC }\end{array}$ & 143 & мн061296 & 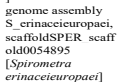 & $2 e-33$ \\
\hline Unigene_36687 & $\left(\mathrm{TCG}_{\mathrm{s}}\right.$ & $\begin{array}{l}F: \text { (HEX)TGCGCATCTCTCAGCATCAT } \\
R: \text { GATGGAGAGCTCTGAGGCAC }\end{array}$ & 254 & Мно61292 & $\begin{array}{l}\text { erinaceieuropaei] } \\
\text { nucleolintike } \\
\text { LOCC1034975s } \\
\text { [Cucumis melo] }\end{array}$ & $9 \mathrm{c}-22$ \\
\hline Unigene_30801 & $(\mathrm{AG})_{14}$ & $\begin{array}{l}F:(\text { TAMRA)TCATAATGCAACTTGACTCGATG } \\
R: \text { TCCTGGACGGTTCAGTGC }\end{array}$ & 172 & MH158508 & $\begin{array}{l}\text { Uncharacterized } \\
\text { LOCIOC698374 } \\
\text { [Brassica oleracea }\end{array}$ & $3 c-07$ \\
\hline Unigene_ 88986 & $\left(\mathrm{TC}_{3}\right.$ & $\begin{array}{l}F: \text { (FAM)GCATTTGCTGGTGTTGCTTG } \\
R: \text { GGTGGTGGGTGAAAGGGAAT }\end{array}$ & 182 & мно661298 & $\begin{array}{l}\text { clone CM43 } \\
\text { microstellite } \\
\text { sequence [Chilina } \\
\text { dombeimal }\end{array}$ & $3 c-04$ \\
\hline Unigene_16056 & $(\mathrm{AT})_{10}$ & $\begin{array}{l}\text { F: (TAMRA)CGTCTGAGAGAGAAAGGAAAGC } \\
R: \text { GTCCATTTCTGCTTTGGAGC }\end{array}$ & 231 & MH158509 & 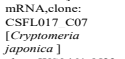 & $7 e-81$ \\
\hline Unigenc_8850 & $(\mathrm{AT})$ & $\begin{array}{l}F: \text { (FAM)ACAAAAGGAAAAACCAGACGTAA } \\
R: \text { :CCTCACAAGGTAGCGGTTGT }\end{array}$ & 200 & мн061286 & 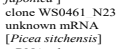 & $4 c-10$ \\
\hline Unigene_26046 & $(\mathrm{AT})_{8}$ & $\begin{array}{l}F: \text { (HEX)CCGCTCCGATTGTTGATGTG } \\
R: \text { ACCTGCCTGCTTGGTAAAATG }\end{array}$ & 245 & Мно61289 & 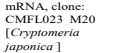 & 0.000 \\
\hline Unigene_20393 & $(\mathrm{AG})_{\Perp}$ & $\begin{array}{l}F: \text { (TAMRA)TGCTCTCCAACAACAACAGC } \\
R: \text { ATTTCATGGCATCTGGTGGT }\end{array}$ & 91 & мH 158510 & 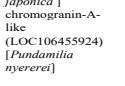 & $2 e-06$ \\
\hline Unigene_942 & $(\mathrm{AT})_{s}$ & $\begin{array}{l}F:(\text { FAM)CTCAGACACAGCTGGCTTCA } \\
R: \text { GGTGAAGATATCTGGAACTTGACG }\end{array}$ & 236 & Мно61284 & 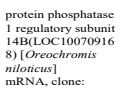 & 0.001 \\
\hline Unigene_50154 & $(\mathrm{AT})_{\mathrm{k}}$ & $\begin{array}{l}F: \text { (HEX)ACTGCAAGAAAA } \\
R: \text { ACCCTTCTGCTGAAAAC }\end{array}$ & 125 & Мноб1295 & [Cryptomeria & 0.000 \\
\hline Unigen___3433 & $(\mathrm{AG})_{19}$ & $\begin{array}{l}F: \text { (TAMRA)CTTTAAGCGTTTCCAGGACAG } \\
R: \text { CTTGGCATGCAGTCTTCTGA }\end{array}$ & 261 & MH1585511 & 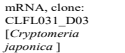 & $60-19$ \\
\hline Unigene__24759 & (CT) & $\begin{array}{l}F: \text { : (FAM)TGTTCCGATGTCTACTGCCG } \\
R: \text { TTTCACTCTCACCAGGTCGC }\end{array}$ & 225 & мно61288 & 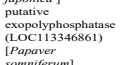 & $1 \mathrm{e}-19$ \\
\hline Unigene_ 37063 & $(\operatorname{AT})_{s}$ & $\begin{array}{l}F: \text { (HEX)TCCATTGAGCACAACCCACT } \\
\text { R: AGCTTTTAGTGGACATGCTTGA }\end{array}$ & 284 & мно61293 & 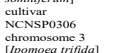 & 0.001 \\
\hline Unigene_106035 & $(A G)_{\mathrm{S}}$ & 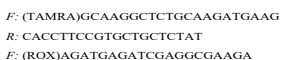 & 187 & мно61299 & 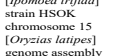 & 0.011 \\
\hline Unigene_16778 & $(\mathrm{AG})_{11}$ & $\begin{array}{l}F:(\text { ROX)AGATGAGATCGAGGCGAAGA } \\
R: \text { CGCATTTATGGATCCCGTTA }\end{array}$ & 280 & MH15: & 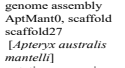 & $4 c-08$ \\
\hline Unigene__34713 & $(\mathrm{GA})_{\mathrm{B}}$ & $\begin{array}{l}F: \text { (HEX)GCAATCTTTGGGCCATGTGG } \\
R: \text { CTAGACCAGCACGCGTAGAG }\end{array}$ & 245 & Мно61290 & 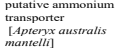 & 0.000 \\
\hline Unigene_45772 & $(\mathrm{AT})_{\mathrm{s}}$ & $\begin{array}{l}F:(\text { HEX)GTGGGTCCAGTGGTTCCAAT } \\
R: \text { GGAGATGTTTGTCACACCCCA }\end{array}$ & 273 & МH061294 & 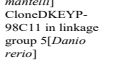 & 0.019 \\
\hline
\end{tabular}


Data analysis

GenAlEx6.5 (Peakall and Smouse, 2012) was used to calculate the following parameters: allele number $(A)$, effective number of alleles $\left(A_{e}\right)$, observed heterozygosity $\left(H_{o}\right)$ and expected heterozygosity $\left(H_{e}\right)$. Microsatellite toolkit (Park, 2001) was used for the calculation of the polymorphic information content (PIC) and a Hardy-Weinberg equilibrium analysis was conducted in Genepop (http://www.genepop.curtin.edu.au/).

\section{Results and Discussion}

After cDNA library construction, transcriptome sequencing and quality filtering, a total of 106,901 unigenes were acquired with an average length of $872 \mathrm{bp}$. A total of 12,279 SSR motifs were identified in 9,597 unigenes. The SSR frequency, i. e. the ratio of unigenes with SSRs to the total number of unigenes was $8.98 \%$, including 1,892 unigenes that contained more than one SSR region. The distribution frequency of SSR motifs, i. e. the ratio of SSR loci to the total number of unigene was $11.48 \%$, which means that on average there is an SSR every $7.59 \mathrm{~kb}$.

Among the motifs identified, trinucleotide repeats were the most abundant type with a frequency of $43.36 \%$, followed by tetranucleotide $(30.60 \%)$, hexanucleotide $(10.45 \%)$, dinucleotide $(8.67 \%)$ and pentanucleotide repeats (6.92 \%). Among the trinucleotide loci, the AAG/CTT motif was the most abundant, followed by AGG/CCT and AGA/TCT.

The number of alleles $(A)$ varied from one to eight in each population, the effective number of alleles $\left(A_{e}\right)$ ranged from $A_{e}=1.00$ to 5.97 , the observed heterozygosity $\left(H_{o}\right)$ from $H_{o}=$ 0.00 to 1.00 , and the expected heterozygosity $\left(H_{e}\right)$ from $H_{e}=$ 0.00 to 0.83 . The polymorphic information content $(P I C)$ varied between loci from $P I C=0.0120$ to 0.7188 , with an average of 0.4001 over all 23 loci (Table 2). Fifteen loci deviated from Hardy-Weinberg equilibrium (HWE) in at least one of the three populations and three (Unigene_24035, Unigene_51014, Unigene_2343) showed significant deviation in all assayed populations (HWE, $\mathrm{P}<0.05$; Table 2) which might be due to the presence of null alleles. However, two out of these three loci (Unigene_51014, Unigene_2343; Table 2) exhibited an excess of heterozygotes in all populations compared to what is expected under HWE which is unlikely to be caused by null alleles. A possible explanation could be associative overdominance or that these loci do not behave in a strictly neutral way (transcriptome data) and are under selective pressure. However, the deviations from HWE could also be due to chance.
Table 2.

Genetic diversity in threeChinese Glyptostrobus pensilis populations based on the 23 polymorphic microsatellite markers.

\begin{tabular}{|c|c|c|c|c|c|c|c|c|c|c|c|c|c|c|}
\hline & \multicolumn{4}{|c|}{$\mathrm{DM}(\mathrm{N}=33)$} & \multicolumn{4}{|c|}{$\operatorname{PISL}(N=29)$} & \multicolumn{4}{|c|}{ GZHN(N=21) } & \multicolumn{2}{|c|}{ Total $(V=83)$} \\
\hline & $A$ & $A_{e}$ & $H_{0}$ & $H_{t}$ & $A$ & $\overline{A_{t}}$ & $H_{0}$ & $H_{t}$ & $A$ & $A_{t}$ & $H_{0}$ & $H_{e}$ & $A$ & PIC \\
\hline unigene 6272 & 4 & 2.177 & 0.300 & $541^{\circ}$ & 3 & 1.110 & 103 & 099 & 3 & 1.706 & 0.333 & .414 & 5 & 0.4820 \\
\hline 240 & 2 & 1.502 & 0.000 & 0.334 & 4 & 2.722 & 0.069 & $0.633^{\circ}$ & 3 & 2.014 & 0.000 & $0.500^{\circ}$ & 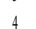 & 0.4789 \\
\hline 15 & 4 & 1.250 & 0.094 & $0.200^{\circ}$ & 3 & 319 & 0.276 & 242 & 3 & 2.228 & 0.200 & $0.551^{\circ}$ & 5 & 0.3206 \\
\hline e 622 & 2 & 1.067 & 0.065 & 0.062 & 2 & 1.035 & 0.034 & .034 & 2 & 1.049 & 0.048 & 0.046 & 2 & 0.3576 \\
\hline unigene 35432 & 1 & 1.000 & 0.000 & 0.0000 & 2 & 1.035 & 0.034 & 0.034 & 2 & 1.100 & 0.000 & $0.091^{\circ}$ & 3 & 0.0357 \\
\hline unigene 3959 & 5 & 2.580 & 0.448 & 0.61 & 2 & 1.039 & 0.038 & & 5 & 3.653 & 0.650 & 0.726 & 7 & 0.7108 \\
\hline 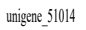 & 5 & 3.372 & 0.970 & 0.703 & 3 & 2.448 & 1.000 & $0.592^{\prime}$ & 4 & 3.000 & 0.952 & $0.66^{\prime}$ & 6 & 9929 \\
\hline unigene_ 36687 & 1 & 1.000 & 0.000 & 0.00 & 2 & 1.890 & 0.759 & $0.471^{\prime}$ & 2 & 1.049 & 0.048 & 0.046 & 2 & 0.2140 \\
\hline ne_ 30801 & 8 & 4.787 & 0.576 & $0.791^{\circ}$ & 2 & 1.071 & 0.069 & 0.067 & 7 & 5.970 & 0.850 & $0.833^{\circ}$ & 9 & 0.7188 \\
\hline ę요 88 & 2 & 1.031 & 0.030 & 0.03 & 1 & 1.000 & 0.000 & 000 & 1 & 1.000 & 0.000 & 0.000 & 2 & 0.0120 \\
\hline unigene 16 & 4 & 1.213 & 0.125 & 0.175 & 3 & 1.764 & 0.500 & 0.433 & 4 & 2.427 & 0.667 & 0.588 & 5 & 0.4588 \\
\hline unigene__ & 1 & 1.000 & 0.000 & 0.000 & 4 & 2.184 & 0.862 & $0.542^{\prime}$ & 1 & 1.000 & 0.000 & 0.000 & 4 & 0.4706 \\
\hline unigene 2t & 3 & 2.068 & 0.697 & 0.517 & 3 & 1.761 & 0.517 & $0.432^{\circ}$ & 3 & 1.537 & 0.429 & 0.349 & 3 & 0.4339 \\
\hline unigene_2 2 & 2 & 1.695 & 0.333 & 0.410 & 2 & 1.109 & 0.10 & 0.098 & 3 & 800 & 0.476 & 0.444 & 3 & 0.3037 \\
\hline unigene 9. & 1 & 1.000 & 0.000 & 0.000 & 2 & 1.708 & 0.586 & 0.414 & 2 & 1.208 & 0.190 & 0.172 & 2 & 0.1966 \\
\hline unigene_5015. & 4 & 2.713 & 0.469 & 0.631 & 2 & 1.035 & 0.034 & $0.034^{\prime}$ & 3 & 2.256 & 0.524 & $0.557^{\prime}$ & 4 & 0.5616 \\
\hline unigene_2343 & 5 & 3.290 & 0.970 & $0.696^{\circ}$ & 2 & 2.000 & 1.000 & $0.500^{\circ}$ & 4 & 3.065 & 1.000 & $0.674^{\circ}$ & 5 & 0.5873 \\
\hline unigene_24759 & 3 & 1.850 & 0.406 & $0.459^{\circ}$ & 1 & 1.000 & 0.000 & 0.000 & 2 & 1.630 & 0.333 & 0.387 & 3 & 0.2750 \\
\hline 37063 & 5 & 2.510 & 0.438 & $0.602^{\circ}$ & 1 & 1.000 & 0.000 & 0.000 & 5 & 139 & 0.810 & $0.681^{\circ}$ & 5 & 0.5720 \\
\hline unigene_106035 & 2 & 1.330 & 0.161 & 0.248 & 2 & 1.035 & 0.034 & 0.034 & 2 & 1.724 & 0.300 & 0.420 & 2 & 0.3748 \\
\hline unigene_167778 & 1 & 1.000 & 0.000 & 0.00 & 2 & 1.665 & 0.552 & 0.400 & 3 & 172 & 0.857 & $0.540^{\circ}$ & 3 & 0.4978 \\
\hline unigene_34713 & 3 & 2.171 & 0.758 & 0.539 & ${ }^{2}$ & 1.998 & 0.966 & $0.499^{\circ}$ & 3 & 2.146 & 0.857 & $0.534^{\circ}$ & 3 & 0.4157 \\
\hline unigene_45772 & 2 & 1.031 & 0.030 & 0.030 & 2 & 1.035 & 0.034 & 0.034 & 2 & 1.100 & 0.095 & 0.091 & 2 & 0.0459 \\
\hline Mean & 3.043 & 1.854 & 0.299 & 0.330 & 2.261 & 1.477 & 0.329 & 0.245 & 3.000 & 2.086 & 0.418 & 0.405 & 9 & 0.4007 \\
\hline
\end{tabular}

"Deviation from Hardy-WVinhers equilibrium $(R<0.05)$

${ }^{2}$ Locality and voucher information are provided in Appendix

Additionally, cross-species amplification of the 23 loci developed in this study was tested in Cryptomeria fortunei, Taxodium distichum, Taxodium ascendens and Cunninghamia lanceolata (Appendix 1) using five individuals each. The number of loci that could be successfully amplified ( $n$ ) and were polymorphic $\left(n_{\text {poly }}\right)$ were as follows: $C$. fortunei $\left(n=22, n_{\text {poly }}=15\right)$, T. distichum $\left(\mathrm{n}=22, \mathrm{n}_{\text {poly }}=17\right)$, Ta. ascendens $\left(\mathrm{n}=21, \mathrm{n}_{\text {poly }}=18\right)$ and $\mathrm{Cu}$. lanceolata $\left(n=16, n_{\text {poly }}=10\right.$ ) (Table 3$)$. Interestingly, out of the four species $\mathrm{Cu}$. lanceolata had the lowest number of polymorphic loci. This may be due to the more distant genetic relationship of $\mathrm{Cu}$. lanceolata which belongs to another subfamily (Cunninghamioideae) compared to Taxodium, Cryptomeria and Glyptostrobus which are all in the same subfamily (Taxodioideae) (Mao et al. 2012).

Table 3

Characterization of 23 SSR markers developed for Glyptostrobus pensilis in four closely related species. ${ }^{\text {a }}$

\begin{tabular}{|c|c|c|c|c|c|c|c|c|c|c|c|c|c|c|c|c|}
\hline \multirow{2}{*}{ Locus } & \multicolumn{4}{|c|}{ Taxodium distichum( $(N=5)$} & \multicolumn{4}{|c|}{ Taxodium ascendens $(N=5)$} & \multicolumn{4}{|c|}{ Conptomeria fortunei $(N=5)$} & \multicolumn{4}{|c|}{ Cunninghamia lanceolatal $(N=5)$} \\
\hline & $A$ & $A_{e}$ & $H_{o}$ & $H_{e}$ & $A$ & $A_{e}$ & $H_{s}$ & $H_{e}$ & $A$ & $A_{e}$ & $H_{o}$ & $H_{c}$ & $A$ & $A_{e}$ & $H_{o}$ & $H_{e}$ \\
\hline unigene 6272 & 2 & 1.724 & 0.600 & 0.420 & 2 & 1.724 & 0.600 & 0.420 & 2 & 1.471 & 0.000 & 0.320 & 2 & 1.724 & 0.600 & 0.420 \\
\hline ne 24035 & 1 & 1.000 & 0.000 & 0.000 & 1 & 1.000 & 0.000 & 0.000 & 1 & 1.000 & 0.000 & 0.000 & & & & \\
\hline unigene 15398 & 1 & 1.000 & 0.000 & 0.000 & 2 & 1.471 & 0.000 & 0.320 & 3 & 2.273 & 0.000 & 0.560 & 2 & 1.923 & 0.000 & 0.480 \\
\hline unigene 62277 & 2 & 1.471 & 0.400 & 0.320 & 1 & 1.000 & 0.000 & 0.000 & 1 & 1.000 & 0.000 & 0.000 & - & - & - & - \\
\hline unigene 35432 & 1 & 1.000 & 0.000 & 0.000 & 3 & 2.381 & 0.200 & 0.580 & 1 & 1.000 & 0.000 & 0.000 & 1 & 1.000 & 0.000 & 0.000 \\
\hline unizene 3959 & 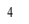 & 2.941 & 0.200 & 0.660 & - & - & & & 2 & 1.471 & 0.400 & 0.320 & & 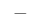 & - & - \\
\hline e 5lt & 3 & 2.273 & 0.800 & 0.560 & 2 & 2.000 & 1.000 & 0.500 & 4 & 3.846 & 1.000 & 0.740 & 4 & 2.941 & 1.000 & 0.660 \\
\hline unigene 36687 & 2 & 1.923 & 0.800 & 0.480 & 3 & 1.852 & 0.600 & 0.460 & 2 & 2.000 & 1.000 & 0.500 & 2 & 1.471 & 0.400 & 0.320 \\
\hline unigene 30801 & 5 & 4.167 & 0.800 & 0.760 & 4 & 2.381 & 0.200 & 0.580 & 4 & 3.846 & 0.600 & 0.740 & - & - & - & - \\
\hline unigene 88986 & 2 & 1.923 & 0.800 & 0.480 & 3 & 1.852 & 0.600 & 0.460 & 2 & 1.724 & 0.600 & 0.420 & 1 & 1.000 & 0.000 & 0.000 \\
\hline unigene 34713 & 4 & 3.333 & 0.600 & 0.700 & 4 & 3.333 & 0.800 & 0.700 & 1 & 1.000 & 0.000 & 0.000 & 3 & 2.273 & 0.800 & 0.560 \\
\hline ene 16. & - & - & - & - & - & - & - & - & 1 & 1.000 & 0.000 & 0.000 & - & - & - & - \\
\hline unigene 16678 & 2 & 1.923 & 0.800 & 0.480 & 2 & 2.000 & 1.000 & 0.500 & 3 & 2.632 & 1.000 & 0.620 & 1 & 1.000 & 0.000 & 0.000 \\
\hline unizene 8850 & 1 & 1.000 & 0.000 & 0.000 & 2 & 1.471 & 0.000 & 0.320 & 2 & 1.923 & 0.000 & 0.480 & 1 & 1.000 & 0.000 & 0.000 \\
\hline unigene 26046 & 4 & 3.333 & 0.800 & 0.700 & 3 & 1.852 & 0.200 & 0.460 & 1 & 1.000 & 0.000 & 0.000 & 3 & 2.632 & 0.600 & 0.620 \\
\hline unigene 20393 & 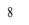 & 7.143 & 1.000 & 0.860 & & 5.556 & 1.000 & 0.820 & 5 & 4.545 & 0.800 & 0.780 & & 2.632 & 100 & 0.620 \\
\hline unigene 9 & 6 & 4.545 & 0.800 & 0.780 & & 3.125 & 0.600 & 0.680 & 3 & 2.381 & 1.000 & 0.580 & & 3.333 & 100 & 0.700 \\
\hline unigene 50154 & 2 & 2.000 & 1.000 & 0.500 & 3 & 1.852 & 0.200 & 0.460 & 2 & 2.000 & 1.000 & 0.500 & 1 & 1.000 & 0.000 & 0.000 \\
\hline uniene 2343 & 5 & 4.545 & 0.200 & 0.780 & 3 & 2.778 & 0.000 & 0.640 & - & - & - & - & - & - & - & - \\
\hline unigene 24 & 1 & 1.000 & 0.000 & 0.00 & & 1.000 & 0.000 & 0.000 & 2 & 1.471 & 0.400 & 0.320 & & 1.000 & 0.000 & 0.000 \\
\hline unigene 37063 & 2 & 1.220 & 0.200 & 0.180 & 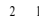 & 1.923 & 0.800 & 0.480 & 1 & 1.000 & 0.000 & 0.000 & & - & - & - \\
\hline unizene 106035 & 2 & 2.000 & 1.000 & 0.500 & 2 & 2.000 & 1.000 & 0.500 & 2 & 2.000 & 1.000 & 0.500 & & 2.000 & 1.000 & 0.500 \\
\hline & & 2.381 & & & & & & & & & & & & .8.46 & & 0.740 \\
\hline
\end{tabular}


In this study we developed 23 polymorphic microsatellite loci which can be used to assess the genetic diversity of $G$. pensilis populations and underpin conservation efforts for this threatened species. One of the 23 loci (Unigene_24035) showed significant deviation from HWE due to an excess of homozygotes in all assayed populations which might be due to the presence of null alleles and should be used with caution. Two loci (Unigene_51014, Unigene_2343) had significantly more heterozygotes than expected. As these loci were developed from transcriptome data, they might linked to regions of the genome which are under selection. If this is the case then Unigene_51014 and Unigene_2343 could potentially be useful to study adaptation. Additionally, the result of our cross-amplification test suggests that the 23 loci might also be useful in genetic studies of related species in Cupressaceae.

\section{Acknowledgements}

This project was supported by forestry industry standard project of China (2014-LY-213). The Royal Botanic Garden Edinburgh is supported by the Scottish Government's Rural and Environment Science and Analytical Services Division.

\section{Data Archiving Statement}

Raw sequencing reads were deposited in the National Center for Biotechnology Information (NCBI) Sequence Read Archive (accession no. PRJNA505868). All primer sequences were uploaded to NCBI (accession no. MH061284-MH1299; MH158506-MH158512; Table 1)
Peakall R, Smouse PE (2012) GenAIEx version 6.5: Genetic analysis in Excel. Population genetic software for teaching and research-An update. Bioinformatics 28:2537-2539. https://doi.org/10.1093/bioinformatics/bts460

Simon SA, Zhai J, Nandety RS, Mccormick KP, Zeng J, Mejia D, Meyers BC (2009) Short-read sequencing technologies for transcriptional analyses. Annual Review of Plant Biology 60:305-333. https://doi.org/10.1146/annurev.arplant.043008.

Tsumura Y, Yoshimura K, Tomaru N, Ohba K (1995) Molecular phylogeny of conifers using RFLP analysis of PCR-amplified specific chloroplast genes. Theoretical and Applied Genetics 91:1222-1236. https://doi.org/10.1007/bf00220933

Thomas P, Lepage BA (2011) The end of an era?: the conservation status of redwoods and other members of the former Taxodiaceae in the 21 st century. Japanese Journal of Historical Botany 19:89-100.

Thomas P, Yang Y, Farjon A, Nguyen D, Liao W (2011) Glyptostrobus pensilis. The IUCN Red List of Threatened Species 2011: e.T32312A9695181. https://doi.org/10.2305/iucn.uk.2011-2.rlts.t32312a9695181.en

Ueno S, Moriguchi Y, Uchiyama K, Ujino-Ihara T, Futamura N, Sakurai T, Shinohara K, Tsumura Y (2012) A second generation framework for the analysis of microsatellites in expressed sequence tags and the development of EST-SSR markers for a conifer, Cryptomeria japonica. BMC Genomics 13:1-16. https://doi.org/10.1186/1471-2164-13-136

Wu ZY (2011) Study on Conservation Biology and Restoration Technique of the Relict Plant Glyptostrobus pensilis [Ph.D.thesis]. Fujian Agriculture and Forestry University, Fujian Province, China.

Wang GT, Wang ZF, Wang RJ, Liang D, Jiang GB (2019) Development of microsatellite markers for a monotypic and globally endangered species, Glyptostrobus pensilis (Cupressaceae). Applications in Plant Sciences 7: e1217. https://doi.org/10.1002/aps3.1217

Xu XH, Li MP (1959) The ecology and geographical distribution of Glyptostrobus pensilis. J. South China Normal Univ. 3:84-99.

Yu YF (1995) Origin evolution and distribution of the Taxodiaceae. Acta Phytotaxon. Sin. 33:362-389.

\section{References}

Averyanov LV, Phan KL, Nguyen TH, Nguyen SK, Nguyen TV, Pham TD (2009) Preliminary observation of native Glyptostrobus pensilis (Taxodiaceae) stands in Vietnam.Taiwania 54:191-212. https://doi.org/10.6165/tai.2009.54(3).191

Li FG, Xia NH (2004) The geographical distribution and cause of threat to Glyptostrobus pensilis (Taxodiaceae). J Trop Subrtop Bot 12:13-20. https://doi.org/10.3969/j.issn.1005-3395.2004.01.003

Li FG, Xia NH (2005) Population structure and genetic diversity of an endangered species, Glyptostrobus pensilis (Cupressaceae). Botanical Bulletin of Academia Sinica 46:155-162. https://doi.org/10.7016/BBAS.200504.0155

LePage BA (2007) The taxonomy and biogeographic history of Glyptostrobus Endlicher (Cupressaceae). Bulletin of the Peabody Museum of Natural History 48: 359-426. https://doi.org/10.3374/0079-032X(2007)48[359:TTABHO]2.0.CO;2

Mao K, Milne Rl, Zhang L, Peng Y, Liu J, Thomas P, Mill RR, Renner SS (2012) Distribution of living cupressaceae reflects the breakup of pangea. Proceedings of the National Academy of Sciences, 109: 7793-7798. https://doi.org/10.1073/pnas.1114319109

Nguyen MT, Vu DD, Bui TTX, Nguyen MD (2013) Genetic variation and population structure in Chinese water pine (Glyptostrobus pensilis): a threatened species. Indian Journal of Biotechnology 12:499-503.

Park SDE (2001) The Excel microsatellite toolkit. Trypanotolerance in west African cattle and the population genetic effects of selection. [Ph.D. thesis]. University of Dublin, Dublin, Ireland. 